\title{
Static and dynamic ionization levels of transition metal-doped zinc chalogenides
}

\author{
Cesar Tablero
}

\begin{abstract}
Transition metal (TM) impurities in semiconductors have a considerable effect on the electronic properties and on the lattice vibrations. The unfilled $d$ shell permits the impurity atoms to exist in a variety of charge states. In this work, the static donor and acceptor ionization energies of $\mathrm{ZnX}: \mathrm{M}$, with $\mathrm{X}=\mathrm{S}$. Se. Te and $\mathrm{M}: \mathrm{Sc}, \mathrm{Ti}, \mathrm{V}$, $\mathrm{Fe}, \mathrm{Co} . \mathrm{Ni}$ are obtained from first principles total energy calculations and compared with experimental results in the literature where they exist. From these results. many of the TM-doped zinc chalogenides have an amphoteric behavior. To analyze the rule of the deep gap levels in both the radiative and non-radiative processes. the dynamic ionization energies are obtained as a function of the inward and outward $\mathrm{M}-\mathrm{X}$ displacements. In many cases, the changes in the mass and the force constants resulting from the substitution of an impurity center for a lattice atom are small. When the charge or the environment of the impurity changes, the electron population tend to remain compensated. As consequence, the changes in the lattice vibrational modes are small.
\end{abstract}

Keywords lonization levels - Electronic structure . Semiconductors - Impurities in semiconductors .

Radiative and non-radiative processes

\section{Introduction}

The transition metal (TM) impurities in semiconductors have attracted much interest as a result of their

C. Tablero $(\bowtie)$

Instituto de Energía Solar, Universidad Politécnica de Madrid, Ciudad Universitaria $s / n, 28040$ Madrid, Spain

e-mail: ctablero@etsit.upm.es technological importance as well as from the viewpoint of basic physics. In many cases, apparently paradoxical results have been attributed to these impurities. Optical absorption spectra indicate features resulting from $d-d$ transitions. indicating that the $d$ electrons are essentially localized. On the other hand, some of the TM has multiple charge states within the gap of the host semiconductor. The positions of the charge state levels allow an easy characterization of the impurity as a donor, an acceptor or with amphoteric behavior. The impurity is (1) a pure donor. when the donor level lies in the gap. but not the acceptor level. (2) a pure acceptor, if the acceptor level lies in the gap, but not the donor level. (3) amphoteric. i.e. the impurity is both a donor and an acceptor. if both donor and acceptor levels are found within the fundamental gap of the host semiconductor. In accordance with this classification, some of the TM impurities are amphoteric. Haldane and Anderson [1] have shown that it is possible under strong hybridization and delocalization of the $d$ electrons with the host.

From the technological viewpoint, the TM impurities are some of the most common contaminants in II-Vl compounds. Because of this dual behavior, the TM impurities present also apparently contradictory results. The role in radiative and non-radiative recombination processes has been discussed for many years. The deep levels introduced by the TM impurity had been considered as a negative effect because of these deep levels act as very effective Shockley. Read and Hall recombination centers $[2,3]$ via a lattice relaxation multiphonon emission mechanism [4, 5].

On the other hand, one of the most important developments of the last decade in broadband diode-pumped lasers was the discovery of the new class of the Cr-doped zinc chalogenides as a broadly tunable continuous wave laser. In particular, both $\mathrm{Cr}: \mathrm{ZnSe}$ and $\mathrm{Cr}: \mathrm{ZnS}$ lasers have 
demonstrated the highest performance at room temperature, continuous wave, tunable, diode pumped and modelocked regimes [6-8]. The Cr-doped laser materials are characterized by high gain and an intrinsically low-lasing threshold, as well as by such remarkable spectroscopic features as the absence of excited state absorption and negligible non-radiative decay at room temperature. The room temperature $\mathrm{Fe}: \mathrm{ZnSe}$ laser has also been reported [912]. The majority of the known vibronically broadened laser transitions in the infrared are quenched at room temperature because of the high probability of multiphonon relaxation processes. The low maximum phonon frequency in chalcogenides leads to a decrease in the non-radiative decay rate and an increase in fluorescence quantum yield.

At the same time, it has been shown that the negative effect of the non-radiative recombination can be avoided for charge redistribution between all the impurities and charge redistribution with the host semiconductor. The former is related to the Mott transition [13, 14]. The cases of the Cr-doped zinc chalogenides have been analyzed theoretically [15-20]. The results show a decrease in the non-radiative recombination, in accordance with the experimental and technological results in lasers.

This paradoxical behavior is also present in solar cell devices. The deep impurities reduce the efficiency of the solar cells by SRH non-radiative recombination. However, if the increase in the density of the impurities responsible for the mid-gap levels is sufficient to form an intermediate band instead of a deep level, then the negative non-radiative recombination is suppressed. In addition. these solar cell devices with an intermediate band partially full have a theoretical limiting efficiency of $63.2 \%$ [21], in contrast to $40.7 \%$ of conventional single-gap solar cells.

From all of the aforementioned, the electron ionization levels and the charge-transfer processes play a significant role in these materials. In particular. TM-doped II-VI semiconductors have recently attracted the attention of researchers as magnetic semiconductors for spintronics and optoelectronics because of their electronic properties. However, the influence of the TM impurities on lattice vibrations of semiconductors has not attracted as much attention. despite their importance in radiative and nonradiative processes. TM impurities can gain charge with respect to the lattice as a result of the change in the number of electrons in the $d$ shell. The charge state of $d$ impurities can change either through the formation of shallow impurity centers or in the photoionization of the impurity by light. In these cases, the Coulomb field of the charged impurity center displaces ions in the nearest environment, mainly via a breathing mode $[4,5,13,14]$, which strains the lattice in this region of the crystal and, thus, may affect its properties. This change may influence, for instance, the radiative and non-radiative processes. Thus, it seems to be of interest to study the properties of these semiconductors with $d$ metal impurities charged relative to the lattice.

In this work, we analyze the role of TM on the ionization energy levels and on both the radiative and non-radiative processes for doped-ZnX, i.e. $Z n X: M$ with $X=S$, Se. Te and $M=S c$. Ti, V, Fe. Co. Ni. The $\mathrm{Cr}$ impurity in this group is not considered because a detailed analysis has been carried out previously [15-20]. First, the position in energy of the donor and the acceptor ionization levels (i.e. the lower and upper Hubbard bands) will be determined and compared with experimental results in the literature. Secondly, the evolution of the ionization levels with respect to the distance $\mathrm{M}-\mathrm{X}$ will be obtained. From these results, the relative influence of radiative and non-radiative processes will be analyzed.

\section{Calculations}

For the TM elements, it is generally accepted that they preferentially occupy cation lattice sites. For this reason. in our calculations with periodic boundary conditions, one $\mathrm{Zn}$ atom in the zincblende structure was replaced with one impurity atom M. Computations based on the densityfunctional theory (DFT) [22] in the local-spin density approximation (LSDA) have been carried out using a super cell containing 64 atomic sites $\left(\mathrm{M}_{y} \mathrm{Zn}_{1-y} \mathrm{X}\right.$ with $y=1 /$ $32=0.03125$ ) with $\mathrm{X}=\mathrm{S} . \mathrm{Se}, \mathrm{Te}$ and $\mathrm{M}=\mathrm{Sc} . \mathrm{Ti}, \mathrm{V}, \mathrm{Fe}, \mathrm{Co}$. Ni. The standard Kohn-Sham (KS) [23] equations are solved self-consistently [24]. For the exchange and correlation term, the LSDA has been used with the PerdewZunger parametrization to the Ceperley-Alder numerical data [25, 26]. The standard Troullier-Martins [27] pseudopotential is adopted and expressed in the KleinmanBylander [28, 29] factorization. The KS orbitals are represented using a linear combination of confined pseudoatomic orbitals [30]. An analysis of the basis set convergence (with size and with the confinement potential for the orbitals) has been carried out using from single-zeta to double-zeta with polarization basis sets for all atoms and varying the number of the special $k$ points in the irreducible Brillouin zone. In all of the results presented in this work, a double-zeta with polarization functions basis set has been used with periodic boundary conditions and 18 special $k$ points in the irreducible Brillouin zone for a 64 -atom cell. Several further corrections to the calculated energies [31] were applied: potential alignment between a charged defect calculation and the perfect host crystal, and spurious interaction of periodic image charges.

As it is well known, a single particle LSDA monoelectronic levels have no rigorous meaning. We. thus. calculate the ionization energies $\varepsilon_{\alpha \%}$. which correspond to the value of the Fermi energy at which the impurity or the 
defect changes from a charge state $\alpha$ to $\alpha-1$ ( $D^{\alpha} \rightleftharpoons D^{\alpha-1}+e$ ) from total energy calculations using the formation energy method [32]. These donor and acceptor energies are equivalent to the ionization energy and the electron affinity of the impurity + host system. Similarly. the donor and acceptor energies of the host semiconductor are the ionization energy (energy required to remove an electron from the top of the valence band (VB) to a position just outside the material. i.e. the vacuum level) and the electron affinity (energy of the bottom of the conduction band (CB) with respect to the vacuum level). respectively. When the impurity takes up a substitutional site in the host semiconductor, the system is neutral, its total energy is $E[0$; $D^{\alpha}$ ] and the formal configuration of the impurity is $D^{\alpha}$. If the system is multiply charged with $-n$ electrons ( $n$ electrons have been removed), the total charge of the system is $n$, its total energy is $E\left[n ; D^{\alpha-n}\right]$ and the formal configuration of the impurity is $D^{\alpha-n}$. The formation energy of this system multiply charged is defined as

$$
\begin{aligned}
\Delta H\left(E_{\mathrm{F}}, n\right) & =E\left(n ; D^{\alpha-n}\right)-E_{\mathrm{H}}-\sum_{i} n_{i} \mu_{i}+n E_{\mathrm{F}} \\
& =E\left(n ; D^{\alpha-n}\right)-E\left(0 ; D^{\alpha}\right)+n E_{\mathrm{F}}
\end{aligned}
$$

where $E_{\mathrm{F}}$ is the Fermi energy, $E_{\mathrm{H}}$ is the total energy for the neutral solid containing only host atoms, $n_{j}$ indicates the number of atoms of type $i$ (host atoms or impurity atoms) that have been added to $\left(n_{i}>0\right)$ or removed from $\left(n_{i}<0\right)$ the solid when the defect or impurity is created, and the $\mu_{i}$ are the corresponding chemical potentials of these species. These chemical potentials represent the energy of the reservoirs with which atoms are being exchanged. We, thus, calculate the transition energies $\varepsilon_{\alpha}$ (donor energy) and $\varepsilon_{\alpha+1}$ (acceptor energy) from total energy calculations as $\varepsilon_{\alpha}=$ $E\left(0 ; D^{\alpha}\right)-E\left(1 ; D^{\alpha-1}\right)$ and $\varepsilon_{\alpha+1}=E\left(-1 ; D^{\alpha+1}\right)-E\left(0 ; D^{\alpha}\right)$ that correspond to the solution of $\Delta H\left(\varepsilon_{\alpha}, 0\right)=\Delta H\left(\varepsilon_{\alpha}, 1\right)$ and $\Delta H\left(\varepsilon_{\alpha+1}, 0\right)=\Delta H\left(\varepsilon_{\alpha+1},-1\right)$, respectively. The previous equations give the absolute energy levels. However, usually they are referred to the bottom of the $C B$ $\left(\varepsilon_{\alpha}^{(\mathrm{CB})}=\varepsilon_{\mathrm{CB}}-\varepsilon_{\alpha}\right)$ or to the top of the VB $\left(\varepsilon_{\alpha+1}^{(\mathrm{VB})}=\varepsilon_{\alpha+1}-\right.$ $\left.\varepsilon_{\mathrm{VB}}\right) . \varepsilon_{\alpha}^{(\mathrm{CB})}$ and $\varepsilon_{\alpha+1}^{(\mathrm{VB})}$ represent the donor and acceptor ionization energies corresponding to the processes $D^{\alpha} \rightleftharpoons D^{\alpha-1}+e_{\mathrm{CB}}$ and $D^{\alpha}+e_{\mathrm{VB}} \rightleftharpoons D^{\alpha+1}$, respectively.

To find a coherent description of both defect energies and the band edges, we obtain them both from electron addition and removal calculations, i.e. in a similar way to the energy levels using the Eq. 1 with all $\mu_{i}$ zero. For the host perfect super cell in its neutral state, we take the VB edge to be the ionization energy $E_{\mathrm{H}}(0)-E_{\mathrm{H}}(+1)$, and the $\mathrm{CB}$ edge to be the electron affinity $E_{\mathrm{H}}(-1)-E_{\mathrm{H}}(0)$, where $E_{\mathrm{H}}(n)$ is the ground state energy of the defect-free super cell as a function of the charge $n$ in it. The resulting estimate $E_{\mathrm{H}^{(}}(-1)-2 E_{\mathrm{H}}(0)+E_{\mathrm{H}^{(}}(+1)$ for the band gap is much closer to the experiment than the estimate from the eigenvalues.

Notice that $\varepsilon_{\alpha}$, $\varepsilon_{\mathrm{VB}}$ and $\varepsilon_{\mathrm{CB}}$ energies depend explicitly on the configuration coordinates $(Q)$ and implicitly on the total system charge $n$. For example, $\varepsilon_{\alpha}^{(\mathrm{CB})}=\varepsilon_{\mathrm{CB}}-\varepsilon_{\alpha}=$ $\left[E_{\mathrm{H}}(-1)-E_{\mathrm{H}}(0)\right]-\left[E\left(0 ; D^{\alpha}\right)-E\left(1 ; D^{\alpha-1}\right)\right]$. In a linear approximation $[4,5]$, the energy levels and total energies vary linearly and quadratically with $Q$. respectively.

\section{Results and discussion}

When the TM M substitutes $\mathrm{Zn}$ in the $\mathrm{ZnX}(\mathrm{X}=\mathrm{S}$. Se and Te) host semiconductor. two of its electrons are given to the bonds, thus, forming the deep impurity level $\mathrm{M}^{2+}\left(\mathrm{d}^{n}\right)$. i.e. with an oxidation state $+2: \mathrm{Sc}^{2+}\left(\mathrm{d}^{\mathrm{l}}\right), \mathrm{Ti}^{2+}\left(\mathrm{d}^{2}\right)$, $\mathrm{V}^{2+}\left(\mathrm{d}^{3}\right), \mathrm{Fe}^{2+}\left(\mathrm{d}^{6}\right), \mathrm{Co}^{2+}\left(\mathrm{d}^{7}\right)$ and $\mathrm{Ni}^{2+}\left(\mathrm{d}^{8}\right)$. Starting from the lattice neutral charge state +2 , donor type $\left(\mathrm{M}^{2+} / \mathrm{M}^{3+}\right)$ as well as acceptor type $\left(\mathrm{M}^{2+} / \mathrm{M}^{+}\right)$conversions are conceivable. It implies the possibility of amphoteric behavior. The donor and acceptor levels (i.e. the lower and upper Hubbard bands) correspond to the transformation of the doubly ionized magnetic ions $\mathrm{M}^{2+}$ into $\mathrm{M}^{3+}$ and into $\mathrm{M}^{1+}$ ions in their ground states, respectively.

The fivefold degenerate $d$ states of TM impurity are split by the crystalline field into doubly degenerate $e_{d}$ states $\left(d_{z^{2}}\right.$ and $\left.d_{x^{2}-y^{2}}\right)$ and threefold degenerate $t_{d}$ states $\left(d_{x v}, d_{x z}\right.$ and $d_{v z}$ ) in the tetrahedral coordination. The electronic configurations of the M-TM at the tetrahedral substitutional site are $\mathrm{Sc}^{2+}\left(e_{d}^{1} t_{d}^{0}\right) . \mathrm{Ti}^{2+}\left(e_{d}^{2} t_{d}^{0}\right), \mathrm{V}^{2+}\left(e_{d}^{2} t_{d}^{1}\right), \mathrm{Fe}^{2+}\left(e_{d t}^{3} t_{d}^{3}\right)$. $\mathrm{Co}^{2+}\left(e_{d}^{3} t_{d}^{4}\right)$ and $\mathrm{Ni}^{2+}\left(e_{d}^{4} t_{d}^{4}\right)$. The crystal wave functions with $t$ symmetry are formed by the combination of the $t_{d}$ and by the states with $t$ symmetry of the host $\left(t_{\mathrm{H}}\right)$; $t^{(i)} \sim \alpha_{i} t_{d}+\beta_{i} t_{\mathrm{H}}$. These $t_{\mathrm{H}}$ states correspond mainly to the neighboring anions $p-X$ states.

Therefore, the $t_{d}$ states hybridize with the $t_{\mathrm{H}}$ states forming the bonding states $(i=1 \equiv b$ ) and anti-bonding counterparts $(i=2 \equiv a)$. The $t^{(b)}$ bonding states appear in the VB host. and the $t^{(a)}$ anti-bonding states emerge in the bandgap or are in the edge of the VB. On the other hand, the wave functions of the $e_{d}$ symmetry are extended to the interstitial region. Therefore, the hybridization of the $e_{d}$ TM states with the $e_{\mathrm{H}}$ symmetry host states of the VB is weak and $e_{d t}$ states remain as non-bonding states. In addition, the spin interaction splits these groups.

In our calculations, one $\mathrm{Zn}$ atom in the zinc blende structure was replaced with one impurity atom M. By analyzing the density of states, significant hybridization is seen between the $d$ bands of $M$ impurities with the nearest neighbors $\mathrm{X}-p$ bands. It is possible that the impurity's $t$ bands may be unusually localized, as is the case in the Mott oxides. Then, the Hubbard $U$ formalism must be used. Nevertheless, a detailed analysis has shown that the effect 
of the correlation in Cr-doped zinc chalogenides is small [15-20].

\subsection{General comparison with other results}

The optical properties of TM-doped II-VI compounds have been researched for a long time. In most of the semiconductors. TM are well known as deep impurities, generally with multiple charge states, which influence the electrical and optical properties of the host semiconductor. Table I gives a summary of the observed acceptor and donor ionization levels in the literature for the TM chalogenides ZnX:M with $\mathrm{X}=\mathrm{S}$. Se. Te and $\mathrm{M}=\mathrm{Sc}$. Ti, V, Fe. Co, Ni. From this table, many of the TM impurities in chalogenides are amphoteric impurities, i.e. the donor and acceptor energies are localized into the gap of the host semiconductor.

Many of the experimental results on ionization levels in the literature are obtained with photo electron-paramagnetic resonance (EPR) and luminescence analysis. By the amphoteric TM impurities have been verified transitions attributed to $\mathrm{M}^{+1} \stackrel{h v}{\longrightarrow} \mathrm{M}^{+2}+\mathrm{e}_{\mathrm{CB}}$ and $\mathrm{M}^{+2} \stackrel{h v}{\longrightarrow} \mathrm{M}^{+1}+\mathrm{h}_{\mathrm{VB}}$, where $h_{V B}$ is the hole in the VB and $e_{C B}$ the electron in the CB. The estimation of the ionization energy levels is usually from theoretical fits to the EPR results.

Using the methodology described in the calculation section. the donor and acceptor ionization levels have been obtained. To analyze the electronic dynamics as a function of the atomic positions, we have also considered the inward and outward displacement of the nearest neighbors of the $M$ atom. i.e. breathing modes. In this breathing mode. the distances M-X $(d)$ are scaled with respect to the distances of the energy minimum $d_{0}: d=\alpha d_{0}$ (inward relaxation for $0<\alpha<1$, and outward relaxation for $\alpha>1$ ). $d$ is chosen as a generalized coordinate $Q$. Of course, there are many vibrational modes. We have chosen a breathing mode because that the forces constants and the displacement of the equilibrium positions are larger than for other modes. Therefore, in principle, their effect in the lattice dynamic will be larger $[4,5]$.
The changes in the ionization energies with the configuration coordinate $Q$ of the breathing mode are shown in Figs. 1,2 and 3 for $X=S$. Se and Te, respectively. An inward relaxation (reduction in $Q$ ) increases the energy of the ionization levels, and an outward relaxation (increase in $Q$ ) diminishes it. Moreover, an inward (outward) movement decreases (increases) the $M$ contribution to the ionization levels.

From Figs. 1, 2 and 3, the gaps obtained using the total energy calculations described in calculation sections are closer to the experimental than those usually obtained using monoelectronic differences. For the equilibrium positions in the $\mathrm{ZnX}: \mathrm{M}$ the gap $\left(e_{\mathrm{C}}-e_{\mathrm{V}}\right)$ is maximum. The gap from the figures $(3.1,2.4$ and $2.3 \mathrm{eV}$ for $\mathrm{X}=\mathrm{S}, \mathrm{Se}$ and $\mathrm{Te}$. respectively) are compared well with the experimental: 3.7 . 2.8 and $2.4 \mathrm{eV}$. The difference can be well appreciated in the figures because the energy range has the experimental gap of the host semiconductor as the upper limit. Despite this gap underestimation, larger from $\mathrm{S}(16 \%)$ to Te $(4 \%)$, we not have corrected it with a shift as is usually done. Note that the habitual gap underestimation using monoelectronic differences with DFT is around $30-50 \%$.

For $Q-Q_{0}>0$ (outward displacement) or $Q-Q_{0}<0$ (inward displacement) the gap is reduced. The decrease is larger for the outward displacements. The $M$ atom is surrounded for $4 X$ in a first shell and for $12 \mathrm{Zn}$ atoms in a second shell. Therefore, the outward mode causes the $X$ of the first shell to come close to the $\mathrm{Zn}$ atoms of the second shell. As a result, the interaction $\mathrm{X}-\mathrm{Zn}$ increases and the gap decreases.

It would be expected that the equilibrium distances $\mathrm{M}-\mathrm{X}$ increases from the $\mathrm{Sc}$ to $\mathrm{Ni}$, according to the increase in the covalent radius of the TM impurities. This behavior is observed in the figures, where the point situated in the $Q-Q_{0}$ axis indicates the equilibrium position distance $\mathrm{Zn}-\mathrm{X}$ of the host semiconductor $\mathrm{ZnX}$. Nevertheless, the equilibrium distances $M-X$ are slightly smaller than the pure sum of covalent radius. This point will be analyzed in more detail in a later section.

Table 1 Observed acceptor and donor ionization energies (eV) with respect to the VB maximum in TM-doped zinc chalogenides

\begin{tabular}{|c|c|c|c|c|c|c|}
\hline & $\mathrm{Sc}$ & $\mathrm{Ti}$ & $\mathrm{V}$ & $\mathrm{Fe}$ & Co & $\mathrm{Ni}$ \\
\hline$e_{\mathrm{A}}(\mathrm{ZnS}: \mathrm{M})$ & & & $2.97[43]$ & & & $\begin{array}{c}2.46[33], 2.41-2.48 \\
{[35-37], 2.4[47]}\end{array}$ \\
\hline$e_{\mathrm{D}}(\mathrm{ZnS}: \mathrm{M})$ & $3.36[33]$ & & $\begin{array}{l}1.96[33] \\
1.84[43]\end{array}$ & $\begin{array}{l}1.3 \pm 0.3[33] \\
1.41-1.47[39]\end{array}$ & & $0.75[35-37]$ \\
\hline$e_{\mathrm{A}}(\mathrm{ZnSe}: \mathrm{M})$ & & & $1.49[44]$ & & & $\begin{array}{l}1.85[33,38], 1.79[34], \\
1.7[47]\end{array}$ \\
\hline$e_{\mathrm{D}}(\mathrm{ZnSe}: \mathrm{M})$ & $1.76[34]$ & $\begin{array}{l}1.75[33,41], \\
1.55[34], 2.03[42]\end{array}$ & $0.88[44]$ & $1.1-1.3[33,48], 0.89[34]$ & $\begin{array}{l}0.25[33] \\
0.37[34]\end{array}$ & $0.16[33], 0.36[34]$ \\
\hline$e_{\mathrm{A}}(\mathrm{ZnTe}: \mathrm{M})$ & & & $1.16[45,46]$ & $1.75[40]$ & & $0.95[38]$ \\
\hline$e_{\mathrm{D}}(\mathrm{ZnTe}: \mathrm{M})$ & & & $0.84[45,46]$ & & & Into VB \\
\hline
\end{tabular}


Fig. 1 Donor $\left(e_{\mathrm{D}}\right)$, acceptor $\left(e_{\mathrm{A}}\right)$ and $\mathrm{CB}$ edge $\left(e_{\mathrm{C}}\right)$ energies with respect to the VB edge $\left(e_{\mathrm{V}}\right)$ energy as a function of $Q-Q_{0}$, for $\mathrm{ZnX}$ :M with $\mathrm{X}=\mathrm{S}$ and $\mathrm{M}=\mathrm{Sc}$ (a), Ti (b), $\mathrm{V}$ (c), $\mathrm{Fe}$ (d), $\mathrm{Co}$ (e) and $\mathrm{Ni}$ (f). $Q$ is the generalized coordinate and $Q_{0}$ is their value for the energy minimum. The thick point situated on the $Q-Q_{0}$ axis indicates the equilibrium position of the host semiconductor $\mathrm{ZnX}$. The horizontal lines correspond to the results in the literature of the Table 1 (a)

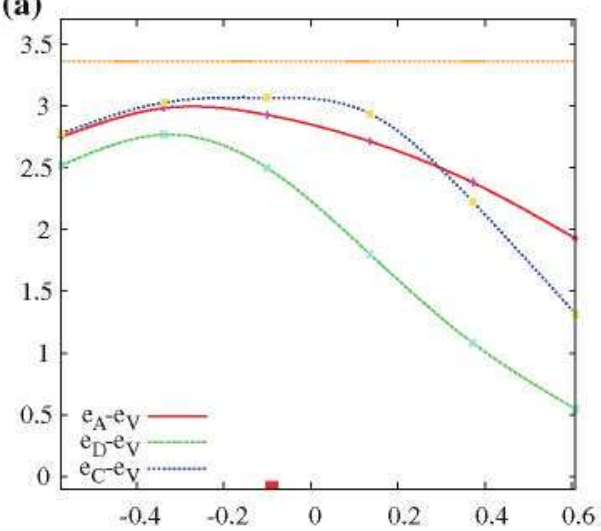

(c)

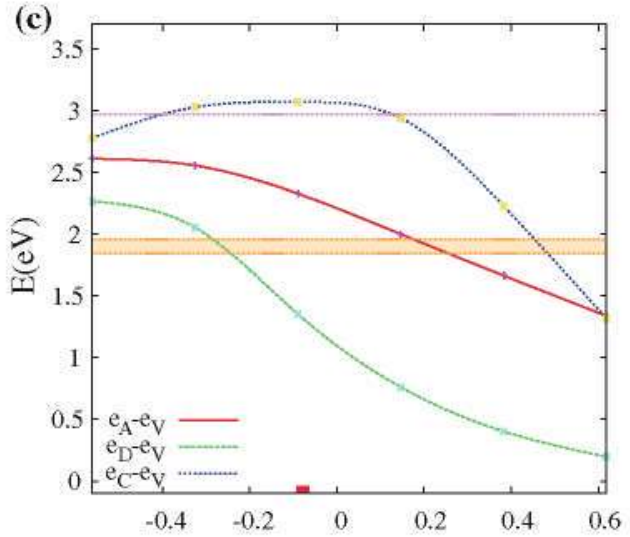

(e)

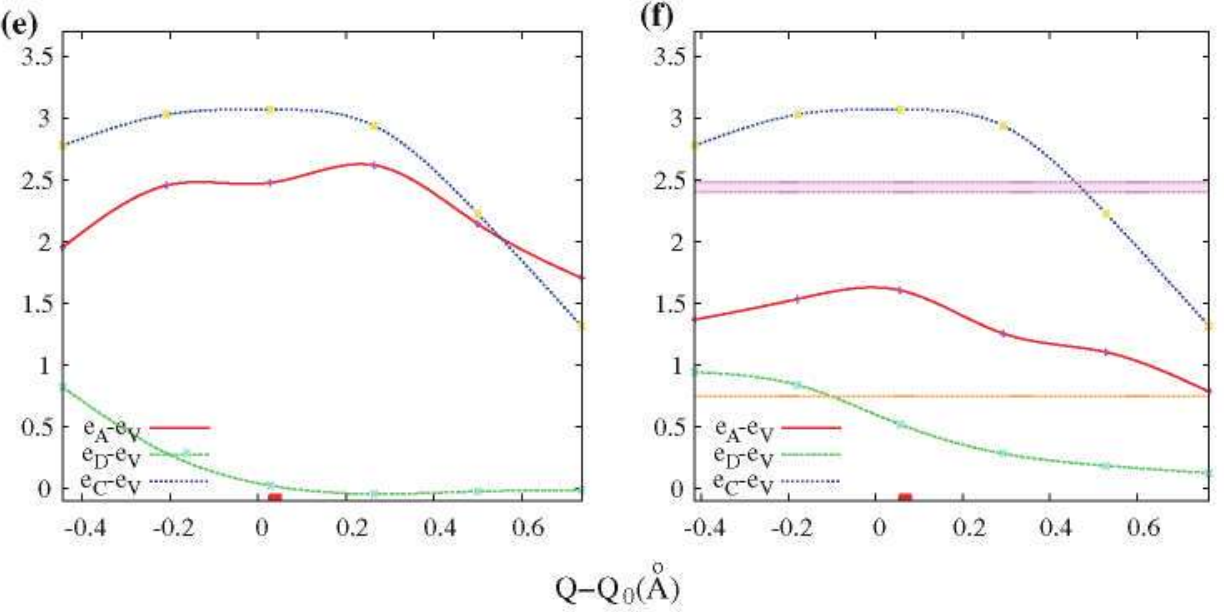

(b)

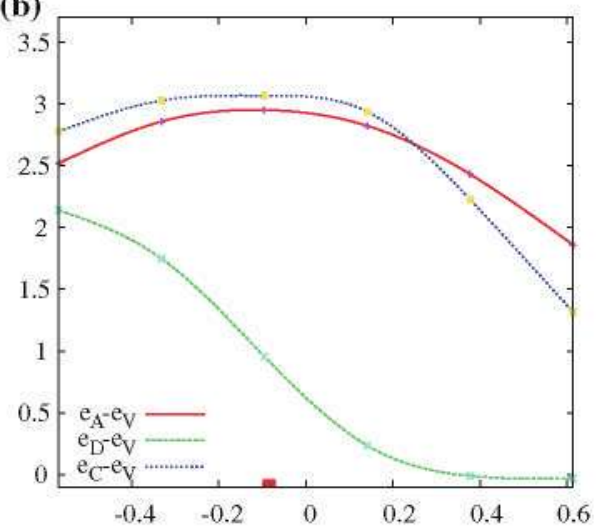

(d)

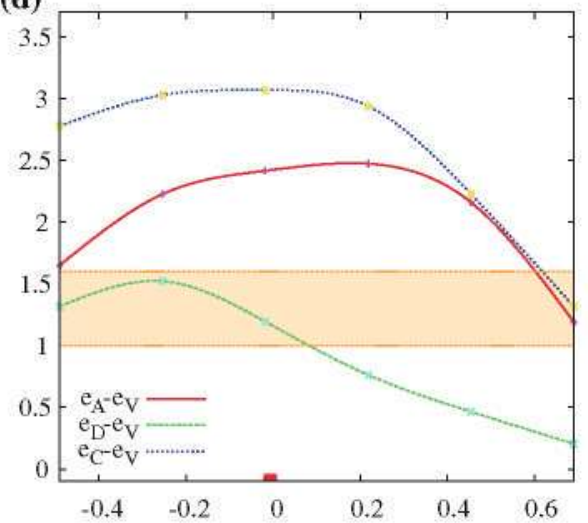

(f)
From Figs. 1, 2 and 3, it is seen that the evolution of the ionization levels with $Q$ is non-linear. It implies an anarmonicity in the curves $D^{\alpha} / D^{\alpha-1}$ and $D^{\alpha} / D^{\alpha+1}$. If the energy curves were quadratic, the energy levels would have a linear dependence with $Q[4,5]$. Note that the ionization energies and the gap decrease towards the right (outward mode) and towards the left (inward mode) of the equilibrium positions. The decrease is larger for the outward mode because of the strong interaction of the first shell of $4 \mathrm{X}$ atoms with the second shell of $12 \mathrm{Zn}$ atoms. This interaction is stronger than the interaction of $M$ with the $4 X$ in the inward mode.

To make comparison with the experimental results easy in Figs. 1, 2 and 3, we have represented the results in the Table 1 with horizontal lines. For the cases where there is experimental dispersion, the horizontal lines have been replaced by rectangles. The results were compared with the results given in Table 1 for the $\mathrm{ZnX}: \mathrm{M}$ ionization levels with $X=S e$ and Te. The comparison is worse for $X=S$, except for the $\mathrm{Fe}$ and $\mathrm{Ni}$ donor levels. The comparison is 
Fig. 2 Same legend as in Fig. 1, but with $\mathrm{X}=\mathrm{Se}$
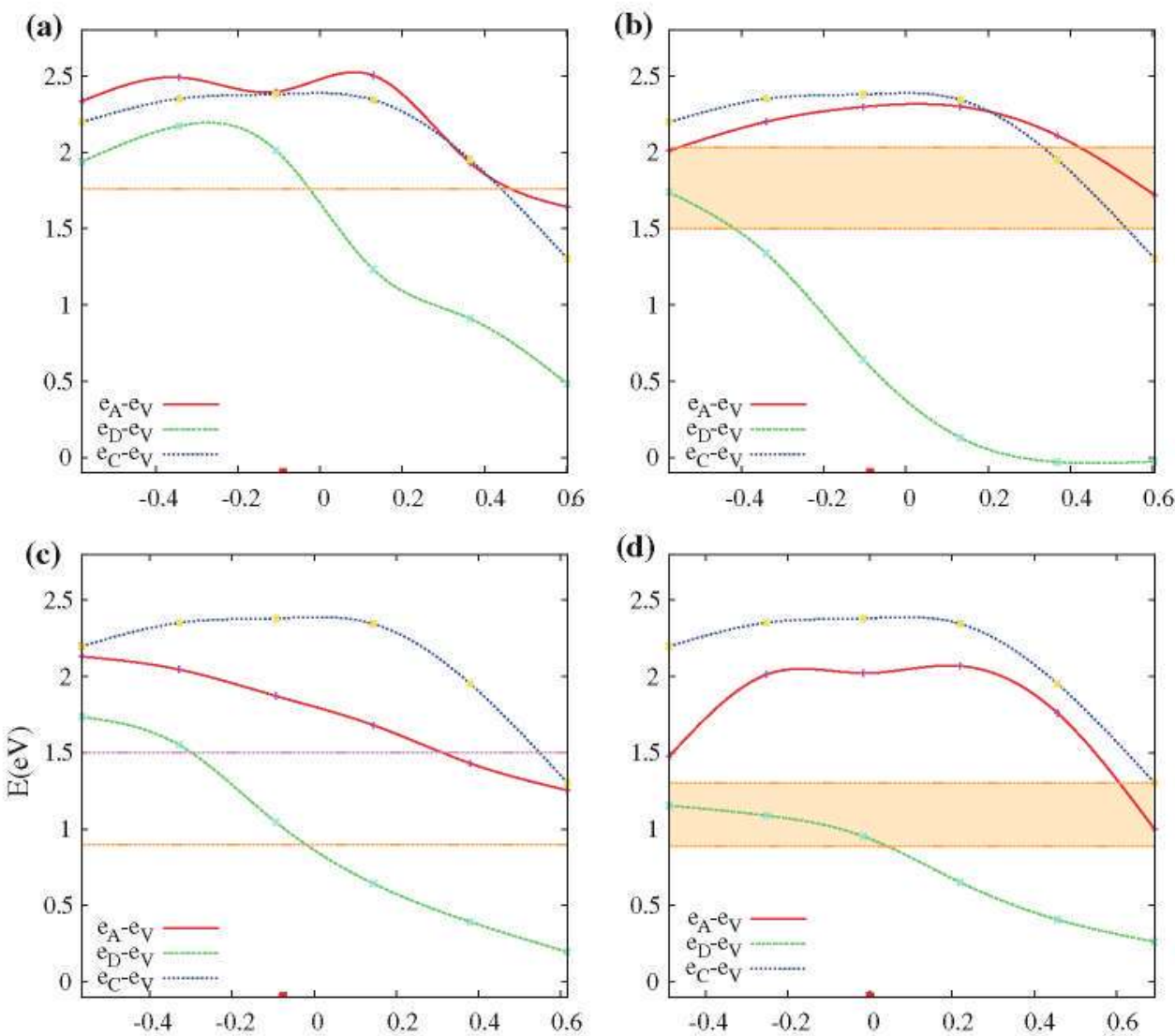

(e)

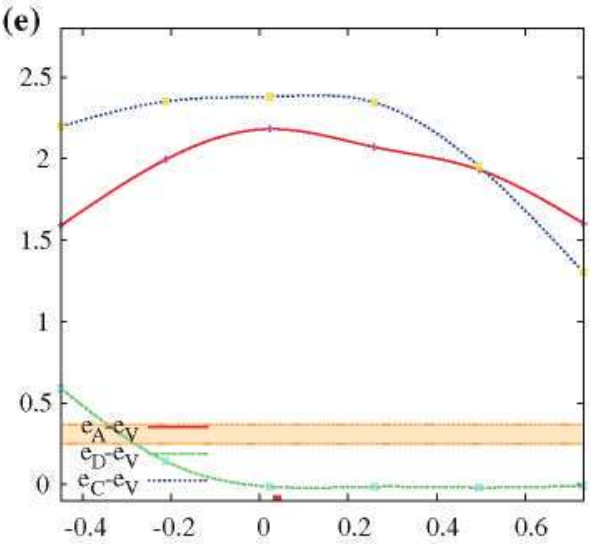

(f)

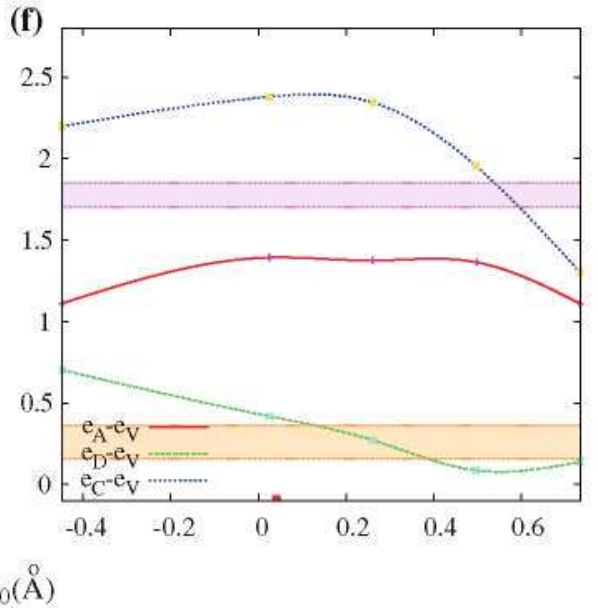

not good for the $\mathrm{V}$ and $\mathrm{Fe}$ acceptor levels. However, these levels are comparable for $\mathrm{X}=\mathrm{Se}$ and Te. Therefore, these differences of the $\mathrm{Fe}$ and $\mathrm{Ni}$ donor levels could be caused by the larger $\mathrm{ZnS}$ gap underestimation.

With respect to the position of the ionization levels in the gap, we observe that the doping with shallow acceptors is necessary to obtain the $p$-type materials as the acceptor levels of all the TM ions lie well above the top of the VB of every zinc chalogenide compound. Moreover, one can see that for all TM (perhaps apart from Co and the ZnTe:Ni) also the donor level lies above the VB and, thus, the appearance of the band holes (even with an additional acceptor doping) is excluded.

The data obtained from the calculations have been fitted to a polynomial of third order around the equilibrium position $Q_{0}: E(Q)=E(0)+(k / 2)\left(Q-Q_{0}\right)^{2}+\left(k_{3} / 6\right)$ $\left(Q-Q_{0}\right)^{3}$ with a root mean deviation lower that $0.01 \mathrm{eV}$. The aforementioned anarmonicity is reflected in the negative value of the $k_{3}$ constant. In agreement with Figs. 1, 2 and 3 and with the covalent radius, there is a monotonic decrease in the distances $\mathrm{M}-\mathrm{X}$. However, the distances $\mathrm{M}-\mathrm{X}$ in $\mathrm{ZnX}: \mathrm{M}$ are larger than the $\mathrm{Zn}-\mathrm{X}$ in the 
Fig. 3 Same legend as in Fig. 1 , but with $X=T e$
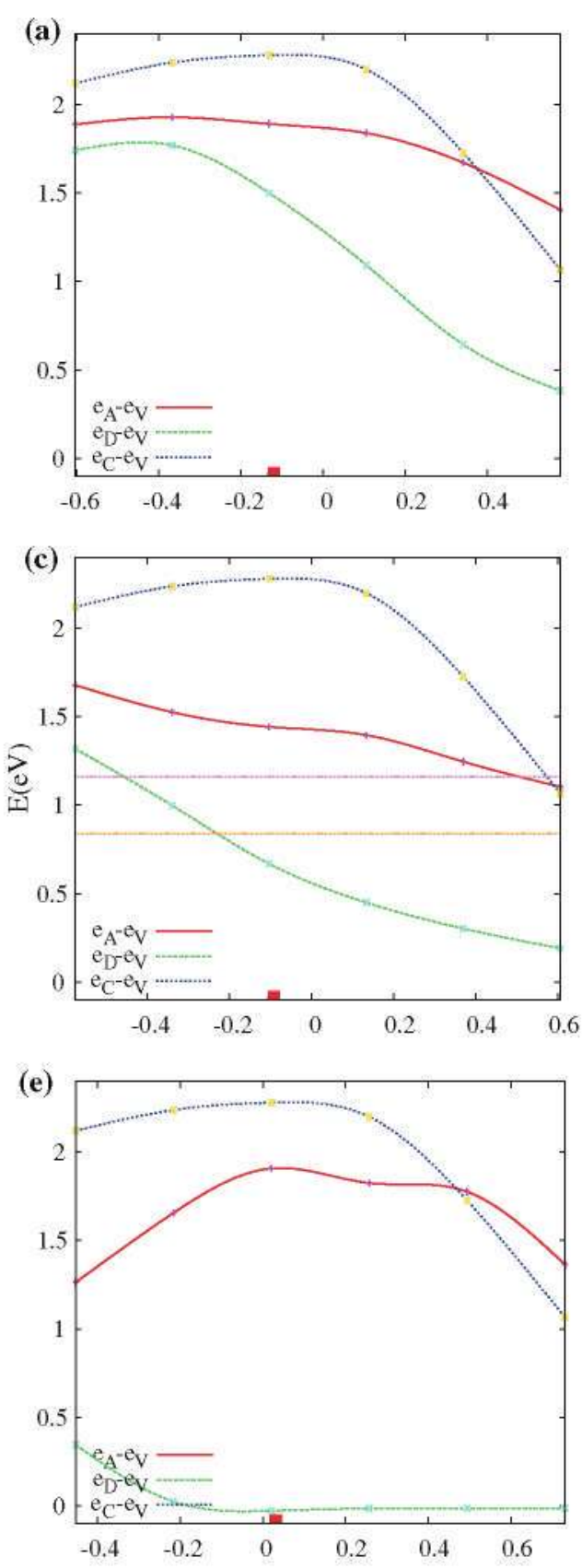

(b)

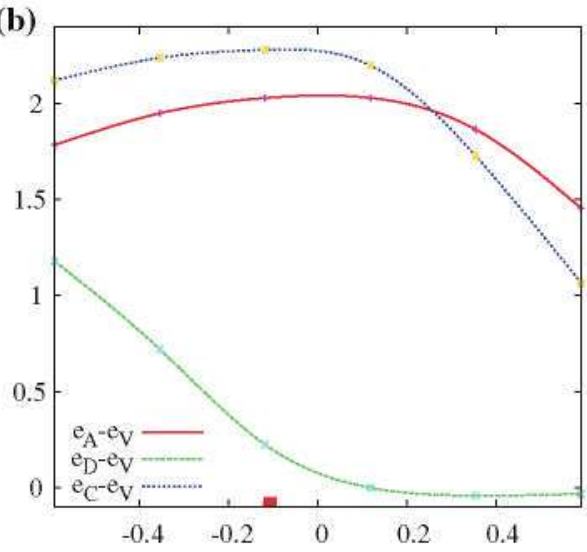

(d)

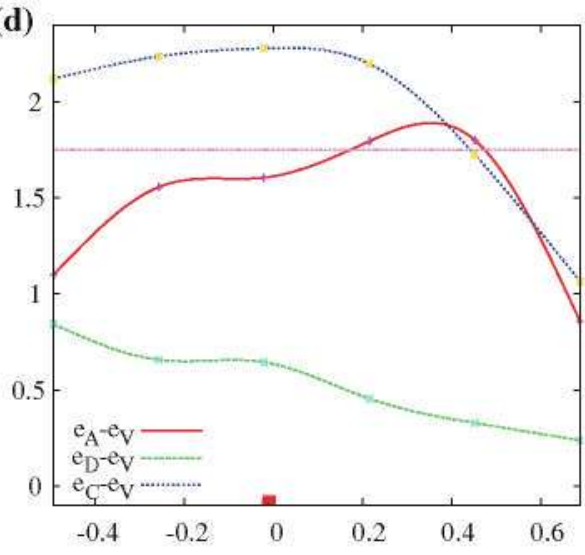

(f)

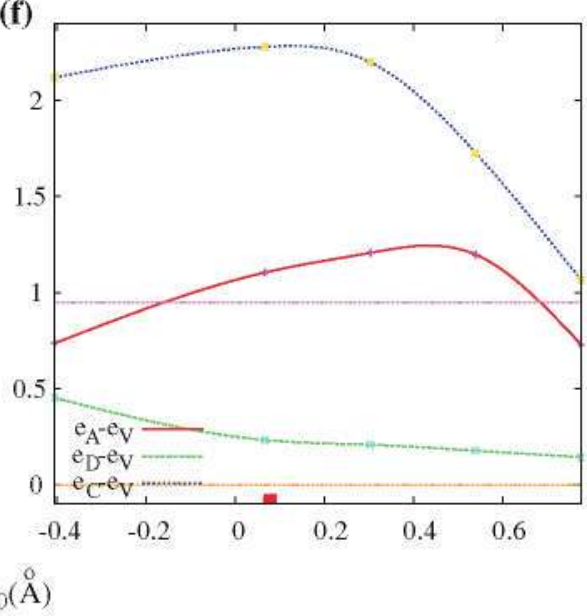

host semiconductor. It indicates that the substitution of the $\mathrm{Zn}$ by the $\mathrm{M}$ atom causes an outward displacement of the $\mathrm{X}$ nearest neighbors. This displacement is larger for the $\mathrm{Cr}, \mathrm{V}$ and $\mathrm{Fe}$.

As a consequence of the increase in the $M-X$ distances with respect to the $\mathrm{Zn}-\mathrm{X}$ distances in the host semiconductor, the bonding $\mathrm{M}-\mathrm{X}$ will be weaker than the $\mathrm{Zn}-\mathrm{X}$. This fact is reflected in the harmonic force constant $k_{2}$. In all cases, $k_{2}(\mathrm{ZnX}: \mathrm{M})<k_{2}(\mathrm{ZnX})$, and the lower values correspond to $\mathrm{ZnS}: \mathrm{Cr}, \mathrm{ZnSe}: \mathrm{Se}, \mathrm{ZnS}: \mathrm{V}$,
$\mathrm{ZnSe}: \mathrm{V}, \mathrm{ZnSe}: \mathrm{Fe}$ and $\mathrm{ZnS}: \mathrm{Ni}$. This aspect could be important in the non-radiative recombination because to smaller force constant smaller will be the probability of a non-radiative transition $[4,5]$. A lower force constant indicates flatter curves in a configuration diagram (total energy vs. $Q$ ). Then, the probability of crossing between the curves of the host and of the impurity will be reduced. This results are in according with the reported $\mathrm{ZnS}: \mathrm{Cr}, \mathrm{ZnSe}: \mathrm{Se}$ and $\mathrm{ZnSe}: F e$ lasers with negligible nonradiative recombination [6-12]. 


\subsection{Energy curves}

As has been previously mentioned. some of the ionization energies have been obtained experimentally from the $\mathrm{M}^{+1} \stackrel{h v}{\longrightarrow} \mathrm{M}^{+2}+\mathrm{e}_{\mathrm{CB}}$ and the $\mathrm{M}^{+2} \stackrel{h v}{\longrightarrow} \mathrm{M}^{+1}+\mathrm{h}_{\mathrm{vB}}$ radiative transitions. Note that in a diagram of energy versus configuration coordinates the radiative processes correspond to vertical transitions. whereas the non-radiative processes correspond to horizontal transitions. These transitions are directly related to the two step mechanism of the nonradiative recombination $[4,5,13,14]$. Using the impurity configuration, $D^{\alpha}$ generically instead of the oxidation state $\mathrm{M}^{+\beta}\left(D^{\alpha+1}, D^{\alpha}\right.$ and $D^{\alpha-1}$ correspond with $\mathrm{M}^{+1}, \mathrm{M}^{+2}$ and $\mathrm{M}^{+3}$. respectively, where $\alpha$ depends on the configuration of the TM impurity), the recombination of an electron from the $\mathrm{CB}$ to the VB is the two step mechanism $D^{\alpha}+$ $e_{\mathrm{CB}} \rightleftharpoons D^{\alpha+1} \rightleftharpoons D^{\alpha}+e_{\mathrm{VB}}$.

Of course. if the total energy of the $D^{\alpha+1}$ is closer to the $D^{\alpha}+e_{\mathrm{VB}}$ total energy (i.e. the acceptor energy closer to the VB), the step $D^{\alpha+1} \rightleftharpoons D^{\alpha}+e_{\mathrm{VB}}$ will be fast, whereas the step $D^{\alpha}+e_{\mathrm{CB}} \rightleftharpoons D^{\alpha+1}$ will be slow. Therefore, the impurity will be a classic acceptor with the acceptor energy closer to the VB. Nevertheless, with deep levels in the gap the two steps must be considered simultaneously. Also, a similar two step mechanism via deep donor level must be considered: $D^{\alpha-1}+e_{\mathrm{CB}} \rightleftharpoons D^{\alpha} \rightleftharpoons D^{\alpha-1}+e_{\mathrm{vB}}$. Note that the non-radiative recombination (horizontal transition) will succeed near the crossing point of the curves. On the other hand, the radiative transitions are verticals.

Using the methodology described in the calculation section, we have obtained the energy curves, as a function of the generalized coordinate $Q$, of $D^{\alpha}+e_{\mathrm{CB}}, D^{\alpha+1}$, $D^{\alpha}+e_{\mathrm{VB} .} D^{\alpha-1}+e_{\mathrm{CB}}, D^{\alpha}$ and $D^{\alpha-1}+e_{\mathrm{VB}}$ systems shown in Figs. 4.5 and 6. From these figures, the anarmonicity of the aforementioned configuration energy curves is clear. According to Figs. 1. 2 and 3, the TM impurities with more deep ionization levels correspond to the $D^{\alpha+1 / \alpha}$ curves localized more symmetrically between the curves $D^{\alpha / \alpha-1}+e_{\mathrm{VB}}$ and $D^{\alpha / \alpha-1}+e_{\mathrm{CB}}$.

The changes in the equilibrium positions $(<0.1 \AA)$ and in the force constants $\left(<5 \mathrm{eV} \AA^{-2}\right)$ resulting from the substitution of an impurity center for a lattice atom are small, and therefore, the changes in the lattice vibrational modes induced by a $d$ impurity are insignificant. One of the reasons for this behavior is the charge density distribution not only for the $\mathrm{Zn}-\mathrm{X}$ atoms, but also for the $\mathrm{M}-\mathrm{X}$ pairs. In some cases as the $\mathrm{Cr}$, $\mathrm{Ni}$ and $\mathrm{Fe}$, the $\mathrm{TM}$ probably donates as many electrons for the formation of chemical bonds as $\mathrm{Zn}$ does in the host semiconductor. The $d$ states, however. can hybridize with the $p$ states of the anions, thus, providing an additional possibility of electronic density redistribution between the $\mathrm{M}$ and $\mathrm{X}$ atoms. This redistribution is a consequence of the contribution of $d$ states to the VB and to the states in the gap. The former have a bonding character. whereas the latter has a non-bonding character. When the charge state of the $M$ impurity is changed by interaction with the light (i.e. when an electron transfers from the VB to the impurity or from the impurity to the $\mathrm{CB}$ ). the excess charge can also undergo redistribution through the hybridization of the M- $d$ states with the $p$ states of the nearest neighboring anions. As a result, the $M$ may have an effective negative charge smaller in magnitude than the electronic charge. Therefore, the occurrence of a strong Coulomb interaction which, coupled to substantial hybridization. leads to a strong screening (also called a self-regulation or L'Chatelier mechanism). This tends to keep the electron population of the impurity virtually unchanged when the overall charge of the impurity or the local environment around TM change.

Non-radiative transitions via multiphonon mechanism are caused by the crossing of the energy curves. The probability of crossing between the energy curves is reduced if the curves are parallels. With a parabolic model, the probability of crossing between two energy curves is lower when the differences between the minimums and between the force constants of the energy curves are small. In general. with non-harmonic curves, the parallelism will avoid the crossing of the curves. Therefore, the L'Chatelier mechanism tends to keep parallels the curves $D^{\alpha}+$ $e_{\mathrm{CB}} \rightleftharpoons D^{\alpha+1} \rightleftharpoons D^{\alpha}+e_{\mathrm{VB}}$ and $D^{\alpha-1}+e_{\mathrm{CB}} \rightleftharpoons D^{\alpha} \alpha-1+$ $e_{\mathrm{VB}}$. The breathing mode is the simplest type of localized vibration with spherical symmetry. Therefore, this L'Chatelier mechanism favors the delocalization and the coupling of the breathing mode with other host system modes.

\section{Conclusions}

We have carried out first principle calculations for $\mathrm{ZnX}: \mathrm{M}$ with $X=S$. Se, Te and M:Sc. Ti, V, Fe, Co, Ni to obtain the ionization levels. We have determined the donor and acceptor ionization levels for both the equilibrium positions (static description) and as a function of the generalized coordinate corresponding to a breathing mode (dynamic description). In almost all cases, except for the $\mathrm{Co}$, there are ionization levels in the gaps. These ionization levels are associated with the anti-bonding combination of the unfilled $d-\mathrm{M}$ shell with the anion $p-\mathrm{X}$ orbitals. The bonding combination (lower energy) lies in the VB. This double contribution to the VB and to the deep levels in the gap permits charge redistribution around the impurity. The main effects in the lattice dynamic are a decrease in the force constants and a small change in the equilibrium positions. As a consequence, the crossing of the curves $D^{\alpha}+e_{\mathrm{CB}} \rightleftharpoons D^{\alpha+1} \rightleftharpoons D^{\alpha}+e_{\mathrm{vB}}$ and $D^{\alpha-1}+$ 
(a)
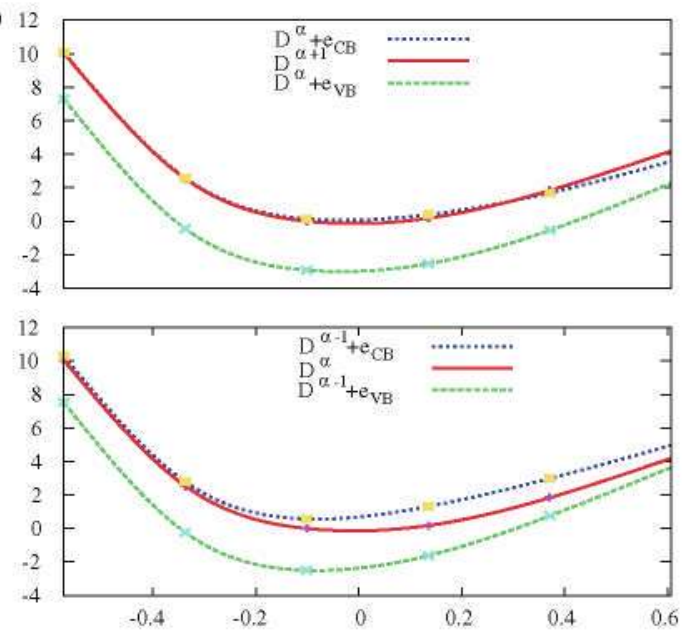

(c)

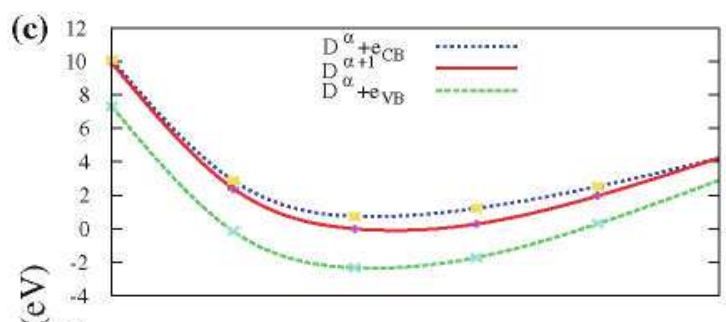

出 12

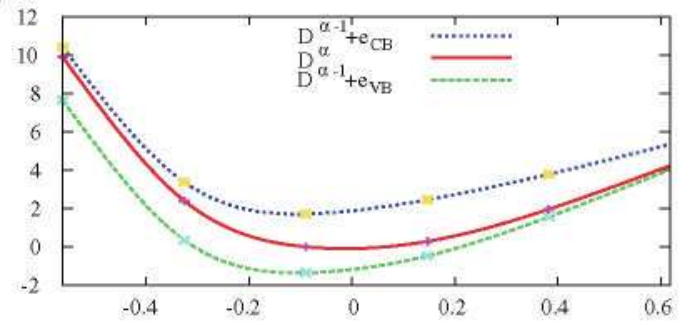

(e)
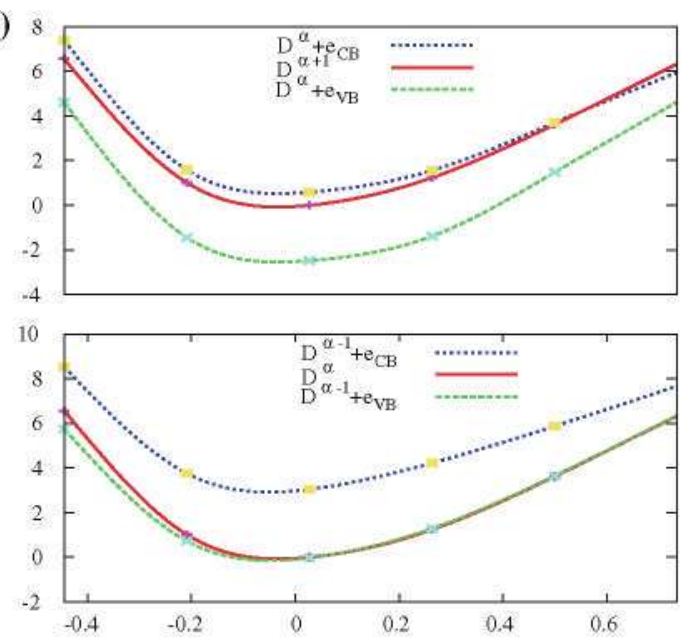

(b)

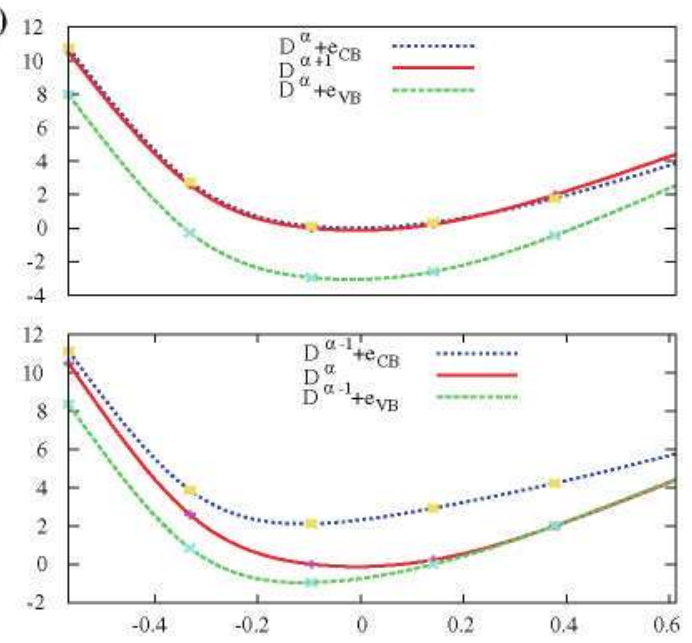

(d)

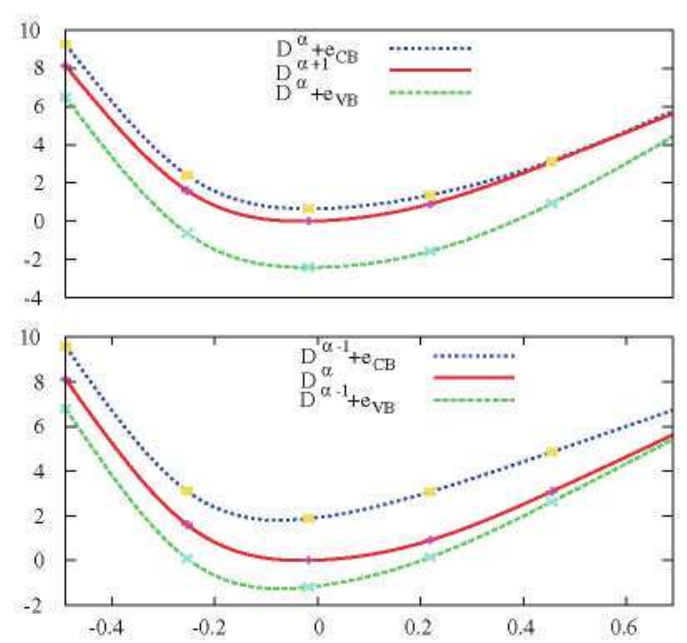

(f)
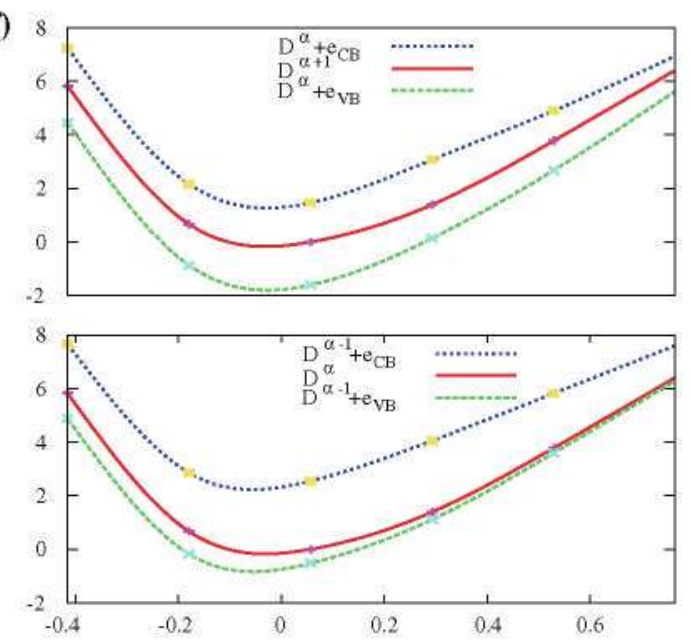

$\mathrm{Q}-\mathrm{Q}_{0}(\AA)$

Fig. 4 Configuration energy curves of the two steps processes $D^{\alpha}+e_{\mathrm{CB}} \rightleftharpoons D^{\alpha+1} \rightleftharpoons D^{\alpha}+e_{\mathrm{VB}} \quad$ (upper panel) and $D^{\alpha-1}+$ $e_{\mathrm{CB}} \rightleftharpoons D^{\alpha} \rightleftharpoons D^{\alpha-1}+e_{\mathrm{VB}}$ (lower panel) for $\mathrm{ZnX}: \mathrm{M}$ with $\mathrm{X}=\mathrm{S}$ and $\mathrm{M}=\mathrm{Sc}$ (a), $\mathrm{Ti}$ (b), $\mathrm{V}$ (c), $\mathrm{Fe}(\mathbf{d}), \mathrm{Co}(\mathbf{e})$ and $\mathrm{Ni}(\mathbf{f}), Q$ is the generalized coordinate and $Q_{0}$ is their value for the energy minimum 
(a)
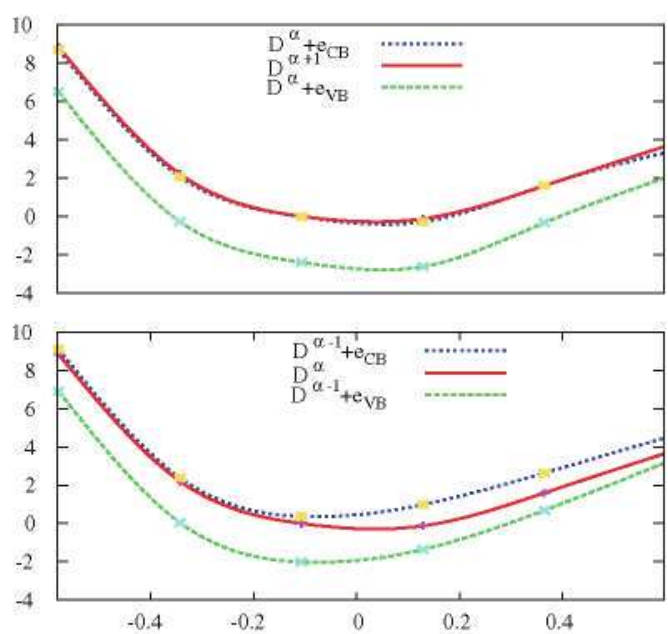

(c)

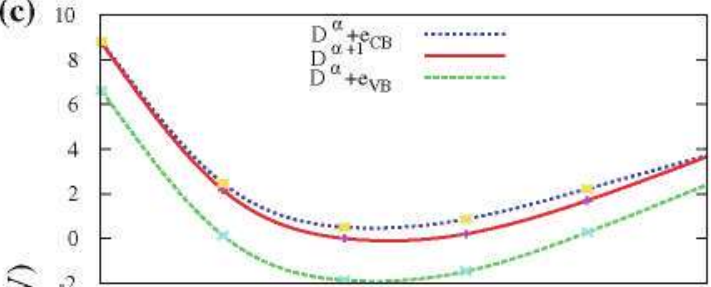

这

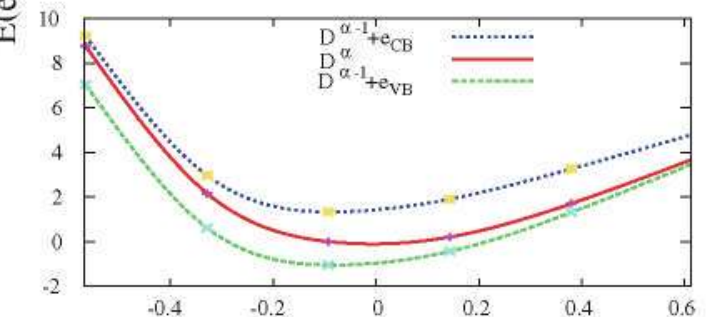

(e)
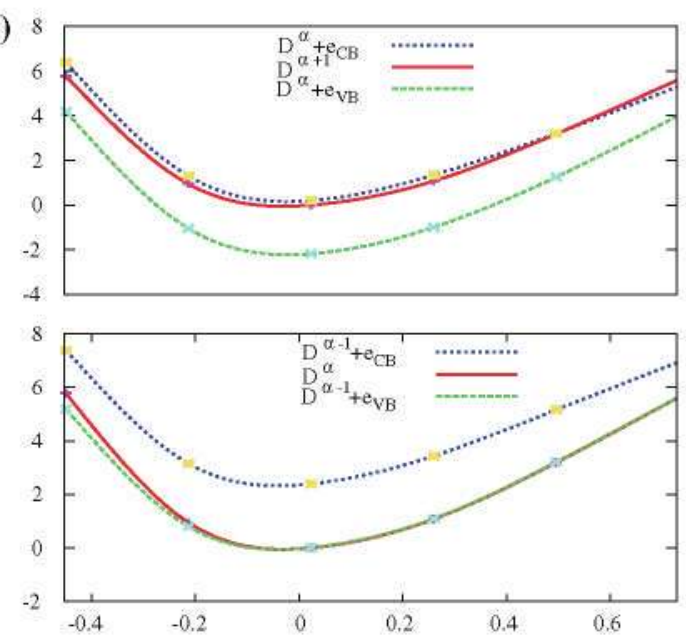

(b)
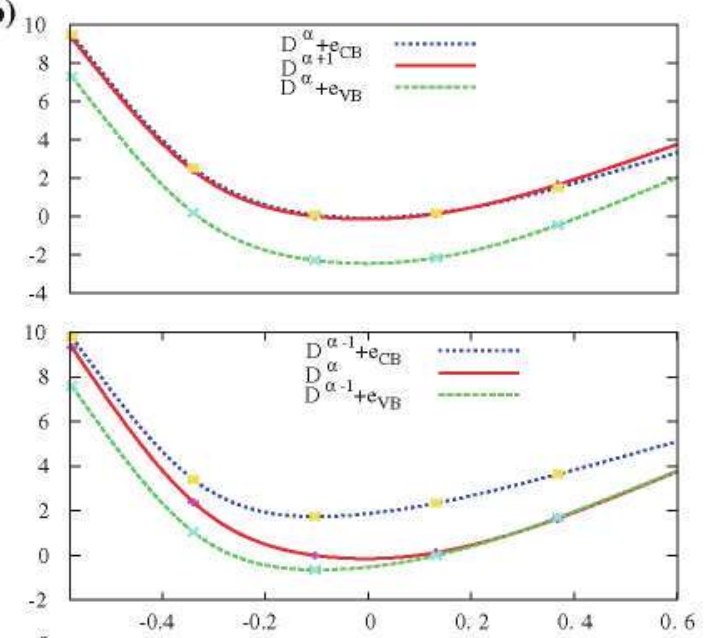

(d)
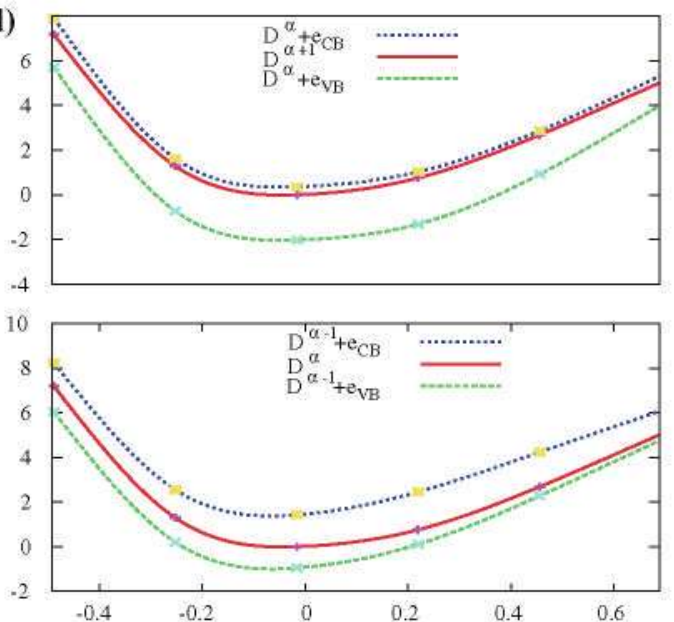

(f)
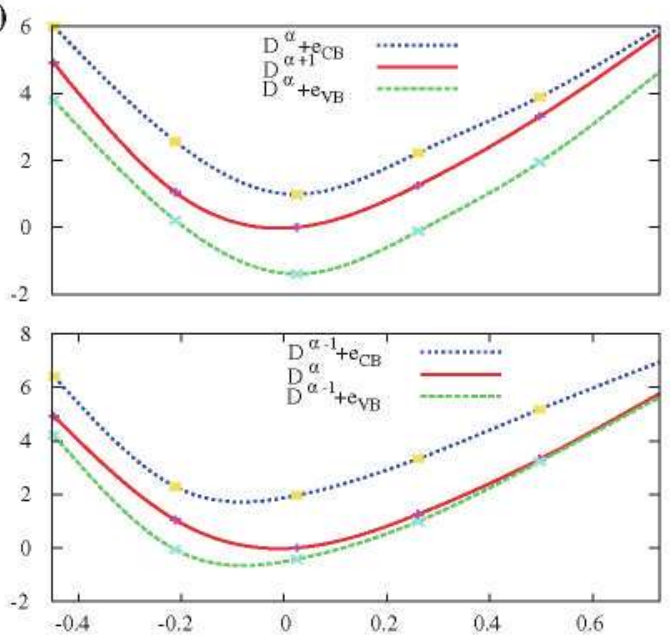

Q-Q $0(\stackrel{\circ}{)}$

Fig. 5 Same legend as in Fig. 4, but with $X=\mathrm{Se}$

$e_{\mathrm{CB}} \rightleftharpoons D^{\alpha} \rightleftharpoons D^{\alpha-1}+e_{\mathrm{VB}}$ is not favorable, reducing the horizontal transitions or the non-radiative recombination, thus improving the emission quantum yield, in accordance with the experimental results. It is particularly important in the $\mathrm{ZnS}: \mathrm{Cr}, \mathrm{ZnSe}: \mathrm{Cr}$, ZnS:V, ZnSe:V, ZnSe:Fe and $\mathrm{ZnSe}: \mathrm{Ni}$. This effect have been corroborated in the $\mathrm{ZnS}: \mathrm{Cr}$, 
(a)
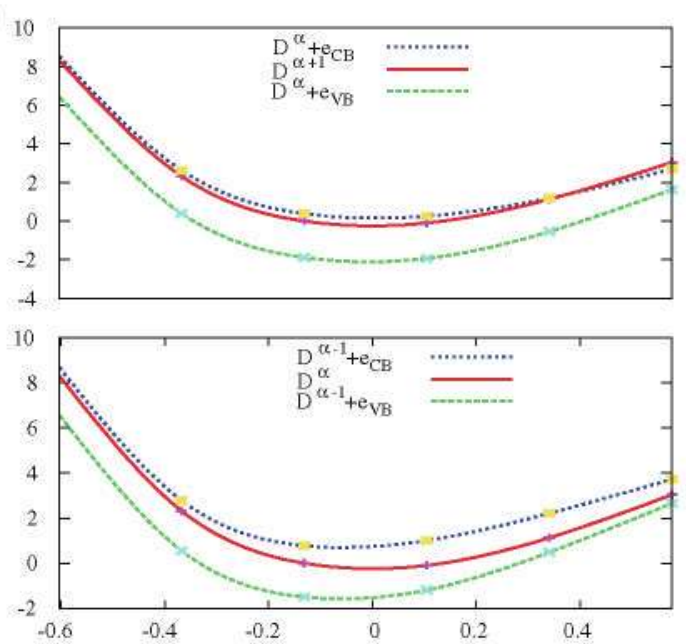

(c)

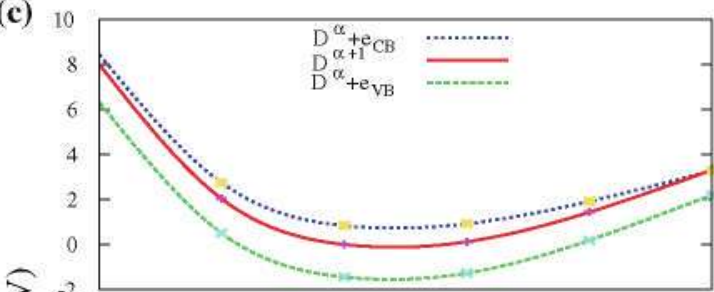

$\frac{3}{1}$

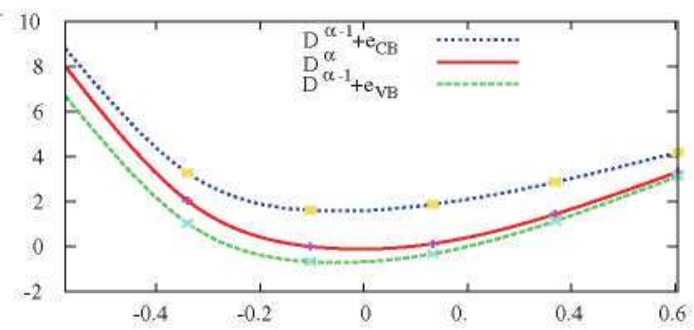

(e)
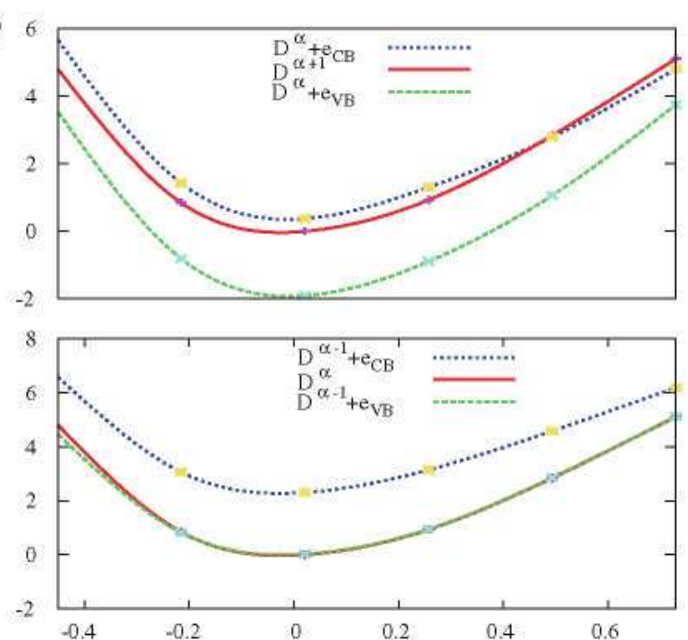

(b)
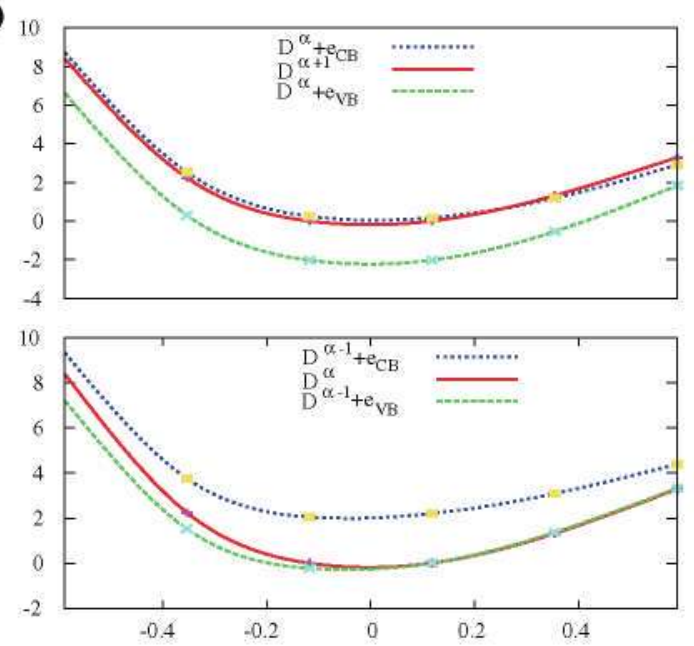

(d) 8

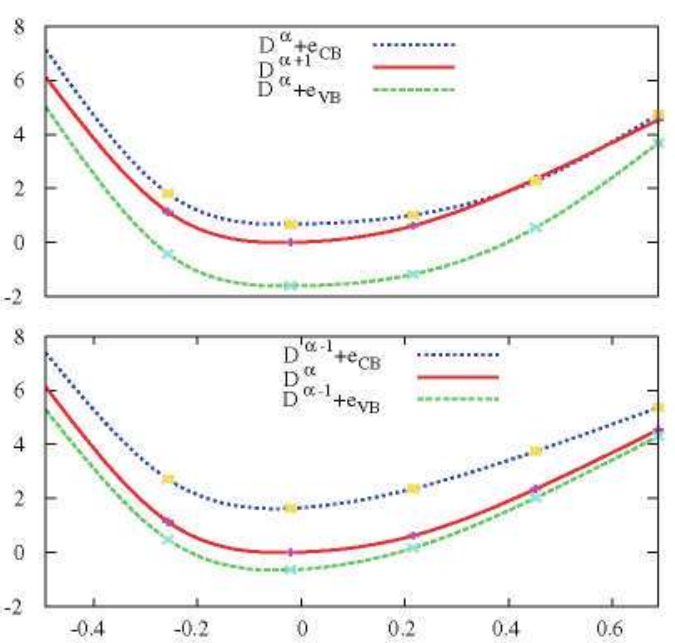

(f) 6

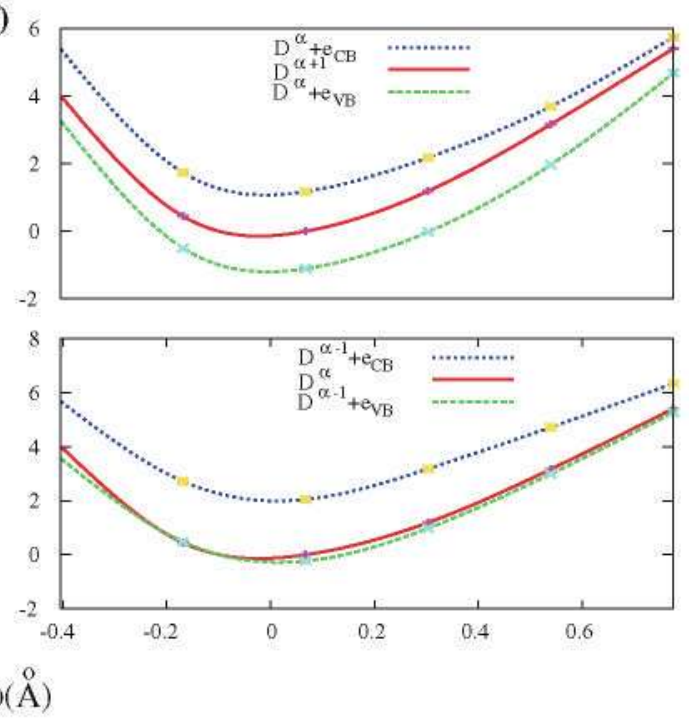

Fig. 6 Same legend as in Fig. 4, but with $X=T e$ 
ZnSe:Cr and ZnSe:Fe lasers where a low probability of the multiphonon relaxation process has been observed.

Acknowledgments This work has been supported by the GENESIS FW project of the National Spanish program CONSOLIDER (CSD2006-0004), by the European Commission through the funding of the project IBPOWER (Ref. N: Grant Agreement 211640), and by La Comunidad de Madrid through the funding of the project NUMANCIA (Ref. N: S-0505/ENE/0310).

\section{References}

1. Haldane FDM, Anderson PW (1976) Phys Rev B 13:2553

2. Shockley W, Read WT (1952) Phys Rev $87: 62$

3. Hall RN (1952) Phys Rev 87:387

4. Lang DV, Henry CH (1975) Phys Rev Lett 35:1525

5. Boer KV (2002) Survey of semiconductor physics. Wiley, London

6. DeLoach LD, Page RH, Wilke GD, Payne SA, Krupke WP (1996) IEEE J Quantum Electron 32:885

7. Page RH, Schaffers KI, DeLoach LD, wilke GD, Patel FD, Tassano JB, Payne SA, Krupke WF, Chen KT, Burger A (1997) IEEE J Quantum Electron 33:609

8. Sorokina IT (2004) Opt Mater 26:395

9. Kernal J, Fedorov VV, Gallian A, Mirov SB (2005) Opt Express 13:10608

10. Adams JJ, Bibeau C, Page RH, Krol DM, Fun LH, Payne SA (1999) Opt Lett 24:1720

11. Akimov VA, Voronov AA, Kozlovskii VI, Korostelin YV, Landman AI, Podmarkov YP, Frolov MP (2004) Quantum Electron 34:912

12. Voronov AA, Kozlovskii VI, Korostelin YV, Landman AI, Podmar'kov YP, Frolov MP (2005) Quantum Electron 35:809

13. Luque A, Martí A. Antolín E. Tablero C (2006) Phys B 382:320

14. Tablero C (2009) Phys B. doi: $10.1016 /$ j.physb. 2009.07 .148

15. Tablero C (2007) Solid State Commun 143:399-402

16. Tablero C (2009) J Chem Phys 130:054903

17. Tablero C (2007) J Chem Phys 126:164703

18. Tablero C (2006) Phys Rev B $74: 195203$
19. Tablero C (2005) J Chem Phys 123:114709

20. Tablero C (2008) Comp Mater Sci 44:303

21. Luque A, Martí A (1997) Phys Rev Lett 78:5014

22. Hohenberg P, Kohn W (1964) Phys Rev B 136:864

23. Kohn W, Sham LJ (1965) Phys Rev 140:Al133-Al138

24. Soler JM. Artacho E、 Gale JD, García A. Junquera J. Ordejon P. Sánchez-Portal D (2002) J Phys Condens Matter 14:2745 and references therein

25. Perdew JP, Zunger A (1981) Phys Rev B 23:5048

26. Ceperley DM, Alder BJ (1980) Phys Rev Lett $45: 566$

27. Troullier N, Martins Л (1991) Phys Rev B 43:1993

28. Kleinman L. Bylander DM (1982) Phys Rev Lett 48:1425

29. Bylander DM, Kleinman L (1990) Phys Rev B 41:907

30. Sankey OF, Niklewski DJ (1989) Phys Rev B 40:3979

31. Persson C, Zhao Y-J, Lany S, Zunger A (2005) Phys Rev B 72:035211

32. Northnp JE, Zhang SB (1993) Phys Rev B 47:6791

33. Fazzio A, Caldas MJ, Zunger A (1984) Phys Rev B 30:3430

34. Langer JM, Delerue C. Lannoo M, Heimrich H (1988) Phys Rev B $38: 7723$

35. Holton WC, Schneider J, Estle TL (1964) Phys Rev 133:A1638

36. Godlewski M, Kaminska M (1980) J Phys C 13:6537

37. Noras JM, Allen JW (1980) J Phys C 13:3511

38. Sokolov VI, Gruzdev NB, Farina IA (2003) Phys Solid State $45: 1638$

39. Skowronski M, Liro Z (1982) J Phys C 15:137

40. Meyer BK. Omling P, Linke H, Salk B, Benz K-W (1992) Appl Phys Lett 61:2911

41. Dziesiaty J, Peka P, Lehr MU, Schulz H-J, Klimakow A (1994) Phys Rev B 49:17011

42. Okada H (1997) Appl Phys Lett 70:1587

43. Biernacki SW, Roussos G, Schulz H-J (1988) J Phys C 21:5615

44. Goetz G, Pohl UW, Schulz H-J (1992) J Phys Condens Matter $4: 8253$

45. Peka P, Lehr MU, Schulz H-J, Pohl UW (1996) Phys Rev B 53:1907

46. Kreissl J, Irmscher K, Peka P, Lehr MU, Schulz H-J, Pohl UW (1996) Phys Rev B 53:1917

47. Surma M. Zakrzewski AJ, Godlewski M (1995) Phys Rev B 52:11879

48. Surma M, Godlewski M, Surkova TP (1994) Phys Rev B 50:8319 